International Mathematical Forum, 1, 2006, no. 25, 1217 - 1226

\title{
Stationary One Point Iteration Algorithm of Arbitrary Order of Convergence for Solving the Distance Equation
}

\author{
M. A. Sharaf \\ Department of Astronomy, Faculty of Science, King Abdul Aziz University ,Jeddah, \\ Saudi Arabia
}

\begin{abstract}
In the present paper, stationary one point iteration algorithm of arbitrary order convergence for solving transcendental equations was given and applied with quintic order for solving the distance equation. Symbolic expressions of the used derivative are also given The accuracy of the numerical results is at least of order $10^{-14}$
\end{abstract}

\section{Introduction}

One of the most crucial pieces of information needed in astronomy is the distance to stars or cosmic groups. For example[1], if the distance $d$ (in parsec) of a star is known as well as its proper motion $\mu$ (in second of arc per year) then one can calculate its tangential velocity $V_{t}$ to the line of sight (in $\mathrm{km}$. per second), which is one of the most useful quantity that could be used for the membership problem for Hyades cluster.Also, having measured the distances to the globular cluster, we can study their distribution in the galaxy[2,3].In moving stellar clusters[e.g the open clusters Hyades and Pleiades] if equatorial coordinates of the vertex and the distance of each member are known, then one can easily determine the velocity of the cluster and also 
the position of its center[4,5] thus the distribution of the cluster's members about this center can be obtained .On the other hand, the determination of distances within our galaxy allows us to calibrate the distance indicators[6,7,8] used to estimate distances outside it. Moreover determining distances would also help astronomers in their quest to understand the size and the age of the universe[9,10],since it would provide an independent estimation of the size of the first steps on the cosmic distance ladder. Consequently it contributes to theories about the origin of the universe Modern observational astronomy has been characterized by an enormous growth in acquisition stimulated by the advent of new technologies in telescopes, detectors and computations. This new astronomical data gives rise to innumerable statistical problems[11]

Recently, a statistical method for cosmic distances determination was developed [12] (hereafter this reference will be referred to as Paper I) .The method depends on transcendental equation involving the error function. Due to the urgent need of accurate cosmic distances because of their important role as mentioned above in brief, the present paper is devoted to develop stationary one point iteration algorithm of arbitrary order of convergence for solving the distance equation. The accuracy of the results is at least of order $10^{-14}$

\section{One-point iteration formulae}

Let $\mathrm{Y}(\mathrm{x})=0$ such that $\mathrm{Y}: \mathbf{R} \rightarrow \mathbf{R}$ smooth map and has a solution $x=\xi$ (say). To construct iterative schemes for solving this equation, some basic definitions are to be recalled as follows:

1. The error in the $\mathrm{k}^{\text {th }}$ iterate is defined as 


$$
\varepsilon_{k}=\xi-x_{k} .
$$

2. If the sequence $\left\{x_{k}\right\}$ converges to $x=\xi$, then

$$
\lim _{k \rightarrow \infty} x_{k}=\xi .
$$

3. If there exists a real number $p \geq 1$ such that

$$
\lim _{i \rightarrow \infty} \frac{\left|x_{i+1}-\xi\right|}{\left|x_{i}-\xi\right|^{p}}=\lim _{i \rightarrow \infty} \frac{\left|\varepsilon_{i+1}\right|}{\left|\varepsilon_{i}\right|^{p}}=K \neq 0,
$$

we say that, the iterative scheme is of order $p$ at $\xi$. The constant $\mathrm{K}$ is called the asymptotic error constant. For $p=1$, the convergence is linear; for $p=2$, the convergence is quadratic; for $p=3,4,5$ the convergence is cubic, quartic and quintic, respectively.

4. One- point iteration formulae are those which use information at only one point. Here, we shall consider only stationary one-point iteration formulae which have the form

$$
x_{i+1}=R\left(x_{i}\right), \quad i=0,1, \ldots
$$

5. The order of one point iteration formulae could be determine either from: (a) The Taylor series of the iteration function $\mathrm{R}\left(x_{n}\right)$ about $\xi$ [e.g. 13]] or from , (b) the Taylor series of the function $Y\left(x_{k+1}\right)$ about $x_{k}$ [e.g. 14]

On the bases of the second approach mentioned above [point ( b)] it is easy to form a class of iterative formulae containing members of all integral orders [ 15] to solve $\mathrm{Y}(\mathrm{x})=0$ as 


$$
x_{i+1}=x_{i}+\delta_{i, m+2} ; \quad \mathrm{i}=0,1,2, \ldots ; \quad \mathrm{m}=0,1,2, \ldots
$$

where

$$
\begin{array}{ll}
\delta_{i, m+2}=\frac{-Y_{i}}{\sum_{j=1}^{m+1}\left(\delta_{i, m+1}\right)^{j-1} Y_{i}^{(j)} / j !} & \delta_{i, 1}=1 ; \forall i \geq 0 . \\
\left.Y_{i}^{(j)} \equiv \frac{d^{j} Y(x)}{d x^{j}}\right|_{x=x_{i}} ; & Y_{i} \equiv Y_{i}^{(0)} .
\end{array}
$$

The convergence order is $m+2$, and is given as

$$
\varepsilon_{i+1}=-\frac{1}{(m+2) !} \frac{Y(\xi)^{(m+2)}}{Y_{i}^{(1)}\left(\xi_{1}\right)} \varepsilon_{i}^{m+2},
$$

where $\xi$ between $x_{i+1}$ and $x_{i}$ and $\xi_{1}$ between $x_{i+1}$ and $\xi$.

\section{The distance equation}

The assumptions upon which the distance equation was derived were[Paper I]:

(1) All the members in a given cosmic group are at the same distance, $r$ parsecs.

(2) The frequency function for the absolute magnitudes of the members

is

$$
\Phi(M)=\frac{1}{\sigma \sqrt{2 \pi}} e^{-\left(M-M_{0}\right)^{2} / 2 \sigma^{2}}
$$

That is the members scatter around a mean absolute magnitude $M_{0}$ in a Gaussian distribution with dispersion $\sigma$. 
(3) The mean apparent magnitude $\bar{m}$ of the members of the cosmic group and the limiting apparent magnitude $m_{1}$ are related through the quantity $\alpha$ where

$$
\alpha=\frac{m_{1}-\bar{m}}{\sigma}
$$

According to the above assumptions, the distance $r$ and the frequency functions $\Psi(m)$ of the apparent magnitude are given respectively by

$$
\begin{gathered}
r=10^{1+\left(m_{1}-M_{0}-\sigma y\right) / 5} \\
\Psi(m)=\frac{1}{\sigma \sqrt{2 \pi}} e^{-\left(m+5-5 \log r-M_{0}\right)^{2} / 2 \sigma^{2}}
\end{gathered}
$$

where $y$ is the solution of the transcendental equation :

$$
\Lambda(y)=y+e^{-y^{2} / 2}\left\{\sqrt{\frac{\pi}{2}}\left[1+\operatorname{erf}\left(\frac{y}{\sqrt{2}}\right)\right]\right\}^{-1}-\alpha=0
$$

and, erf $(z)$ is the error function defined by the integral

$$
\operatorname{erf}(\mathrm{z})=\frac{2}{\sqrt{\pi}} \int_{0}^{z} e^{-t^{2}} \mathrm{~d} t
$$

we call Equation (11) the distance equation.

\section{Numerical developments}

From Equation (7) it is clear that, an iterative scheme for solving Equation (11) includes derivatives of $\Lambda$ as much as the order of the scheme. On the other hand, the higher the order of an iterative scheme , the higher its accuracy and rate of 
convergence will be. Consequently, for the demand of very accurate distance we present in Appendix A the solution of the distance equation using stationary one point iteration algorithm of quintic $(n=5)$ order of convergence. The accuracy of the results are at least $10^{-14}$.

In concluding the present paper, stationary one point iteration algorithm of arbitrary order convergence for solving transcendental equations was given and applied with quintic order for solving the distance equation. The accuracy of the results is at least of order $10^{-14}$. 


\section{References}

1-Robinson,R.,M.: 1985, The Cosmological Distance Ladder, W.H.Freeman and Company New York.

2-Cassisi,S.,De Santis,R and Piersimoni,A.M.:2001,The distance to Galactic globular cluster through RR Lyrae pulsational properties,MNRAS,No.1,342-348.

3-Duncan,D.,Chaboyer,B.,Carney,B,Girard,T.,Latham,D.,Layden,A.,McWilliam,A., Sarajedini,A.and Shao,M.:2001,Anchoring the Population II Distance Scale:Accurate Ages for Globular Clusters and Field Halo Stars.American Astronomical Society Meeting 198\},\#63.09.

4-Sharaf ,M.A.,Bassuny,A.A.and Korany,B.A.:2000,An error controlled method to determine parameteters of moving clusters with application to Hyades, Astrophysical Letter and Communications 40,39-61.

5-Sharaf,M.A.:2003,Relation between the apparent magnitude and the parallax for Hyades stars,New Astronomy 8,645-653

6-Shanks, T :1997,A test of Tully -Fisher distance estimates using Cepheids and SNIa, MNRAS 290,L77-L83 . 
7-Tanvir,N.R.:1997,Cepheids as distance indicators,Conference Paper,Space Telescope Science Institute Series, The Extragalactic Distance Scale,edited by M.Livio,Cambridge University Press,91-112.

8-Brochkhadze,T.M.and Kogoshvili,N.G.:1999,Concerning the errors arising through the use of Tully-Fisher relation for estimation of the Virgo cluster distance, Apj. 42 25-32.

9-Willick,J.A.,Batra,P.:2001,A determination of the Hubble Constant from Cepheid distances and a model of the local peculiar velocity field, Apj. 548 ,2,564-584.

10-Mazumdar,A.,Narasimha,D.:1999,Distance to the Virgo Cluster and estimation of the Hubble Constant.Bulletin of the Astronomical Society of India 27,267.

11-Feigelson,E.D. and Babu,G.,J.(Editors):1992 ,Statistical Challenges in Modern Astronomy,Spriger- Verlag,Berlin

12-Sharaf,M.A.,Issa,I.A. and Saad,A.S.:2003,A method for the determination of cosmic distances,New Astronomy,8,15-21.

13- Ralston, A. and Rabinowitz,P.: 1978, A first Course In Numerical Analysis, Mcgraw-Hill Kogakusha, Ltd. Tokyo. 
14-Danby, J.M.A. and Burkard, T.M.:1983, Celestial Mechanics,31,95.

15-Sharaf, M.A.A. and Sharaf, A.A.: 1998, Celestial Mechanics and Dynamical Astronomy, 69, 331. 
Appendix A: Table I :The solution of the distance equation for some values of $\alpha$

$\begin{array}{llllll}0.15 & \text { 6.372829537 } & 2.15 & 2.105767609 & 4.15 & 4.149927351 \\ 0.25 & \sim 3.524560804 & 2.25 & 2.215233690 & 4.25 & 4.2499522712 \\ 0.35 & \sim 2.2145566459 & 2.35 & 2.322856712 & 4.35 & 4.3499689542 \\ 0.45 & \sim 1.4258406519 & 2.45 & 2.428958493 & 4.45 & 4.4499800063 \\ 0.55 & \sim 0.879495316 & 2.55 & 2.5338102721 & 4.55 & 4.549987252 \\ 0.65 & \sim 0.467097777 & 2.65 & 2.637640487 & 4.65 & 4.6499919525 \\ 0.75 & \sim .1372935987 & 2.75 & 2.7406410727 & 4.75 & 4.7499949704 \\ 0.85 & 0.137601794 & 2.85 & 2.8429726674 & 4.85 & 4.849996888 \\ 0.95 & 0.373949305 & 2.95 & 2.9447690035 & 4.95 & 4.949998093 \\ 1.05 & 0.582106602 & 3.05 & 3.046140684 & 5.05 & 5.0499988436 \\ 1.15 & 0.768991440 & 3.15 & 3.147178482 & 5.15 & 5.149999306 \\ 1.25 & 0.9394264450 & 3.25 & 3.247956246 & 5.25 & 5.2499995872 \\ 1.35 & 1.0968937248 & 3.35 & 3.3485334910 & 5.35 & 5.3499997570 \\ 1.45 & 1.243982052 & 3.45 & 3.4489576856 & 5.45 & 5.4499998584 \\ 1.55 & 1.382664067 & 3.55 & 3.549266286 & 5.55 & 5.5499999183 \\ 1.65 & 1.5144747349 & 3.65 & 3.649488514 & 5.65 & 5.649999953 \\ 1.75 & 1.640629967 & 3.75 & 3.7496469059 & 5.75 & 5.7499999736 \\ 1.85 & 1.762107676 & 3.85 & 3.849758635 & 5.85 & 5.8499999852 \\ 1.95 & 1.8797044884 & 3.95 & 3.9498366318 & 5.95 & 5.949999992 \\ 2.05 & 1.994076267 & 4.05 & 4.049890515 & 6.05 & 6.0499999955\end{array}$

Received: December 5, 2005 